\title{
Vascular complications of prosthetic inter-vertebral discs
}

\author{
Kevin J. Daly · E. Raymond S. Ross • \\ Heather Norris · Charles N. McCollum
}

Received: 1 June 2005/Revised: 2 April 2006/ Accepted: 5 June 2006/Published online: 2 August 2006

(C) Springer-Verlag 2006

\begin{abstract}
Five consecutive cases of prosthetic intervertebral disc displacement with severe vascular complications on revisional surgery are described. The objective of this case report is to warn spinal surgeons that major vascular complications are likely with anterior displacement of inter-vertebral discs. We have not been able to find a previous report on vascular complications associated with anterior displacement of prosthetic inter-vertebral discs. In all five patients the prosthetic disc had eroded into the bifurcation of the inferior vena cava and the left common iliac vein. In three cases the aortic bifurcation was also involved. The fibrosis was so severe that dissecting out the arteries and veins to provide access to the relevant disc proved impossible. Formal division of the left common iliac vein and artery with subsequent repair was our solution. Anterior inter-vertebral disc displacement was associated with severe vascular injury. Preventing anterior disc displacement is essential in disc design. In the event of anterior displacement, disc removal should be planned with a Vascular Surgeon.
\end{abstract}

Keywords Inter-vertebral disc replacement . Artificial inter-vertebral disc $\cdot$ Vascular injury · Disc design · Inter-vertebral arthroplasty

K. J. Daly · C. N. McCollum ( $\varangle)$

Department of Surgery, South Manchester University

Hospital, Manchester, UK

e-mail: cnmcc@manchester.ac.uk

E. R. S. Ross $\cdot$ H. Norris

Department of Orthopaedic Surgery, Hope Hospital,

Salford, UK

\section{Key points}

- Anterior prosthetic disc displacement frequently involves major arteries and veins.

- Removal of an $\mathrm{L}_{45}$ disc prosthesis is associated with vascular injury.

- A Vascular Surgeon should be involved in disc removal.

\section{Introduction}

Replacement of the inter-vertebral disc is designed to relieve symptomatic back pain secondary to disc degeneration by preserving segmental function. A successful artificial disc replacement would avoid the significant long-term problems associated with spinal fusion [9]. We report five consecutive cases of anterior displacement of prosthetic inter-vertebral discs resulting in erosion into the bifurcation of the vena cava and aorta, from a series of only 13 patients. One patient suffered recurrent back pain. These patients had no preoperative vascular symptoms to warn of this vascular involvement and all of these discs were removed in order to prevent further vascular injury. The complications associated with removal are described together with our suggestions on an approach to this challenging surgical problem.

Prosthetic disc replacements (AcroFlex disc, DePuy Acromed, Raynham, MA, USA) were carried out as part of a clinical trial with ethical approval, regulatory approval and with informed patient consent. All patients had incapacitating back pain. Leg pain was not an 
exclusion criteria unless directly attributable to a prolapsed inter-vertebral disc. All patients in this study had magnetic resonance imaging evidence of degenerative disc disease. All patients had an Oswestry disability index (ODI) greater than $30 \%$ and all had discographically established concordant pain at the target level/s. Inclusion criteria, the follow-up protocol and disc design for this study have been described previously [5].

\section{Case 1}

A 33-year-old woman presented with a 5-year history of low back and right leg pain. A two level artificial inter-vertebral disc replacement was performed at $\mathrm{L}_{45}$ and $\mathrm{L}_{5} \mathrm{~S}_{1}$ without complication through an anterior transperitoneal approach $\left(\mathrm{L}_{45} 31.5 \times 43 \times 9 \times 5^{\circ}\right.$ AcroFlex disc and $\mathrm{L}_{5} \mathrm{~S}_{1} 34 \times 46 \times 9 \times 10^{\circ}$ AcroFlex disc) (Fig. 1a-c).

The patient made an uneventful recovery with improved symptoms at 6 weeks. However on X-ray the prosthesis at L45 had moved $5 \mathrm{~mm}$ anteriorly. At 6 months there was further $\mathrm{L}_{45}$ prosthesis displacement; however, mobility was preserved with full lumber flexion and extension at video fluoroscopy (Fig. 1d).

The $\mathrm{L}_{45}$ artificial disc was replaced at 15 months with a size 2 SB Charité III disc containing an $8.5-\mathrm{mm}$ spacer (Waldemar Link GmbH \& Co., Hamburg, Germany). At surgery the disc had stretched and eroded into the left common iliac vein. The distal vein was occluded with thrombus. An injury to the medial wall of the left common iliac artery was repaired with $5 / 0$ Prolene and the occluded vein was oversewn with $4 / 0$ Prolene. This provided access and permitted removal of the disc. Postoperative recovery was complicated by a paralytic ileus only. The patient has an $\mathrm{L}_{5} \mathrm{~S}_{1}$ prosthesis which remains in an acceptable position. She has subsequently become pregnant and had a normal delivery without the need for Caesarian section.

\section{Case 2}

A 40-year-old woman presented with a 3-year history of lumber back pain. She underwent $\mathrm{L}_{45}$ disc replacement with a $29 \times 40 \times 9 \times 10^{\circ}$ AcroFlex disc. This surgery was complicated only by a temporary loss of bladder filling sensation. At 6 week follow-up her back and leg symptoms were significantly improved. Unfortunately at 6 months her back pain had returned and she was now suffering pain and tingling in the left $\mathrm{L}_{5}$ dermatome. On X-ray the prosthesis had moved anteriorly. At 1 year the patient agreed to have the prosthesis removed. At operation the prosthesis was eroding into the left common iliac artery and vein. Both vessels were mobilised with difficulty due to dense scar tissue, the inevitable venous and arterial injuries were repaired with $4 / 0$ Prolene. The disc was replaced with a Stabilis cage (Stryker, UK) containing artificial bone graft which was secured with a $3.2 \times 34 \mathrm{~mm}$ screw and washer. Postoperatively the patient wore class II compression hosiery and was prescribed $40 \mathrm{mg}$ Clexane twice daily. Unfortunately, despite this prophylaxis, she suffered left common iliac artery and iliofemoral deep vein thrombosis. Full anticoagulation was achieved with Tinzaparin and subsequently Warfarin. Swelling is controlled by class II compression hosiery. Transluminal angioplasty has been performed for a stenosis of the left common iliac artery at the site of repair. Her claudication has improved and is now thought to be venous.

\section{Case 3}

A 51-year-old man presented with bilateral leg pain and no motor or sensory loss. He had undergone laminectomy 12 years previously for a disc prolapse. A prosthetic inter-vertebral disc $\left(29 \times 40 \times 9 \times 10^{\circ}\right.$ AcroFlex disc) was placed in the $\mathrm{L}_{45}$ inter-vertebral space, without complication, using an anterior transperitoneal approach. The postoperative X-ray showed the prosthesis to be in an acceptable position, i.e. centrally placed in the disc space on antero-posterior projection and posteriorly placed on the lateral view (Fig. 2a, b). The patient's symptoms were improved but the prosthesis had migrated $2 \mathrm{~mm}$ anteriorly at 6 weeks and $5 \mathrm{~mm}$ by 12 months (Fig. 2c).

At operation the disc had migrated into the left common iliac vein posterior to the aortic bifurcation. A tear was made in the left iliac vein while mobilising these vessels. The left common iliac vein was divided transversely to facilitate removal of the disc. A 14-mm carbon fibre cage filled with iliac crest bone graft was inserted and the iliac vein re-anastomosed with $4 / 0$ Prolene. Postoperative duplex scanning showed the left common iliac vein and artery to be patent. The spine was stabilised with a flat plate and pedicle screws 12 days later (Fig. 2d).

\section{Case 4}

A 47-year-old man presented with lumbar back pain radiating to his thigh and buttock. He underwent $\mathrm{L}_{45}$ 
Fig. 1 Postoperative X-ray with satisfactory placement of $\mathrm{L}_{45}$ and $\mathrm{L}_{5} \mathrm{~S}_{1}$ AcroFlex disc, lateral view (a) AP view (b and c) with anterior disc displacement at 6 months (d)
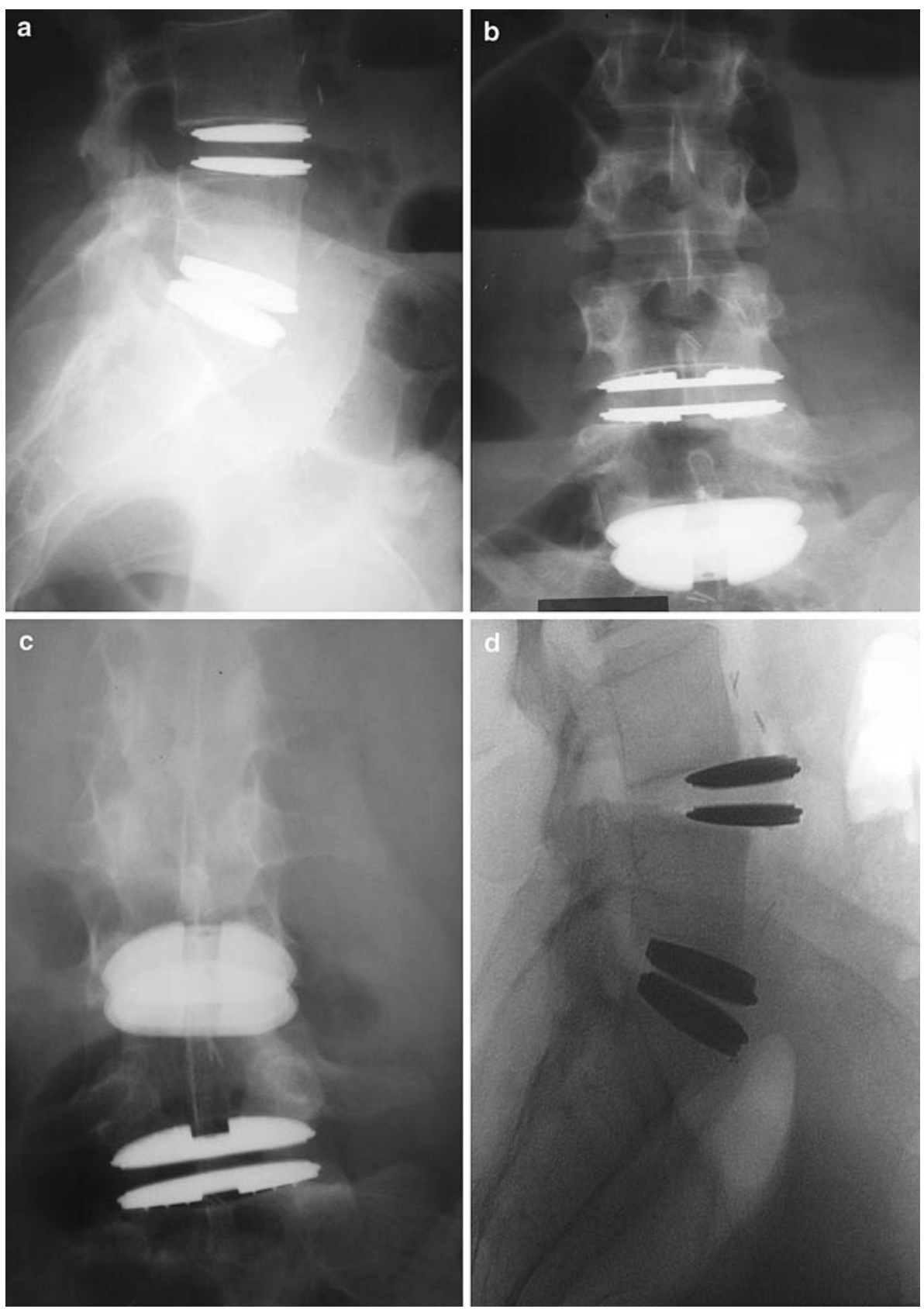

and $\mathrm{L}_{5} \mathrm{~S}_{1}$ disc replacement with a $31.5 \times 43 \times 11^{\circ}$ AcroFlex disc and a $31.5 \times 43 \times 9^{\circ}$ AcroFlex disc respectively without postoperative complication. At 1 month follow-up he was well with no pain.

Anterior displacement of the $\mathrm{L}_{45}$ disc was first seen on plain X-ray at 2 years and the disc was removed 11 months later. Having learned from previous bitter experiences, the vascular surgeon formally divided the left common iliac artery and vein so that there was good access to remove the disc and replace it with a Stabilis cage. The left common iliac artery and vein were then re-anastomosed. Despite this approach the right common iliac vein was injured and required repair. Full deep vein thrombosis prophylaxis was undertaken with pneumatic compression in theatre, peri-operative low molecular weight Heparin, postoperative compression stockings in addition to anticoagulation with Warfarin. Both iliac vessels were widely patent with no thrombus and normal blood flow velocity on duplex imaging at 1 month. Postoperatively he has continued to suffer pain after prolonged standing with occasional sleep disturbance. The $\mathrm{L}_{5} \mathrm{~S}_{1}$ disc was stable on X-ray at 6 months with fusion progressing between $\mathrm{L}_{45}$. 
Fig. 2 Satisfactory placement of $\mathrm{L}_{45}$ AcroFlex disc, lateral view (a) and AP view (b) with anterior displacement at 12 months (c) and subsequent spinal stabilisation with plate and pedicle screws (d)
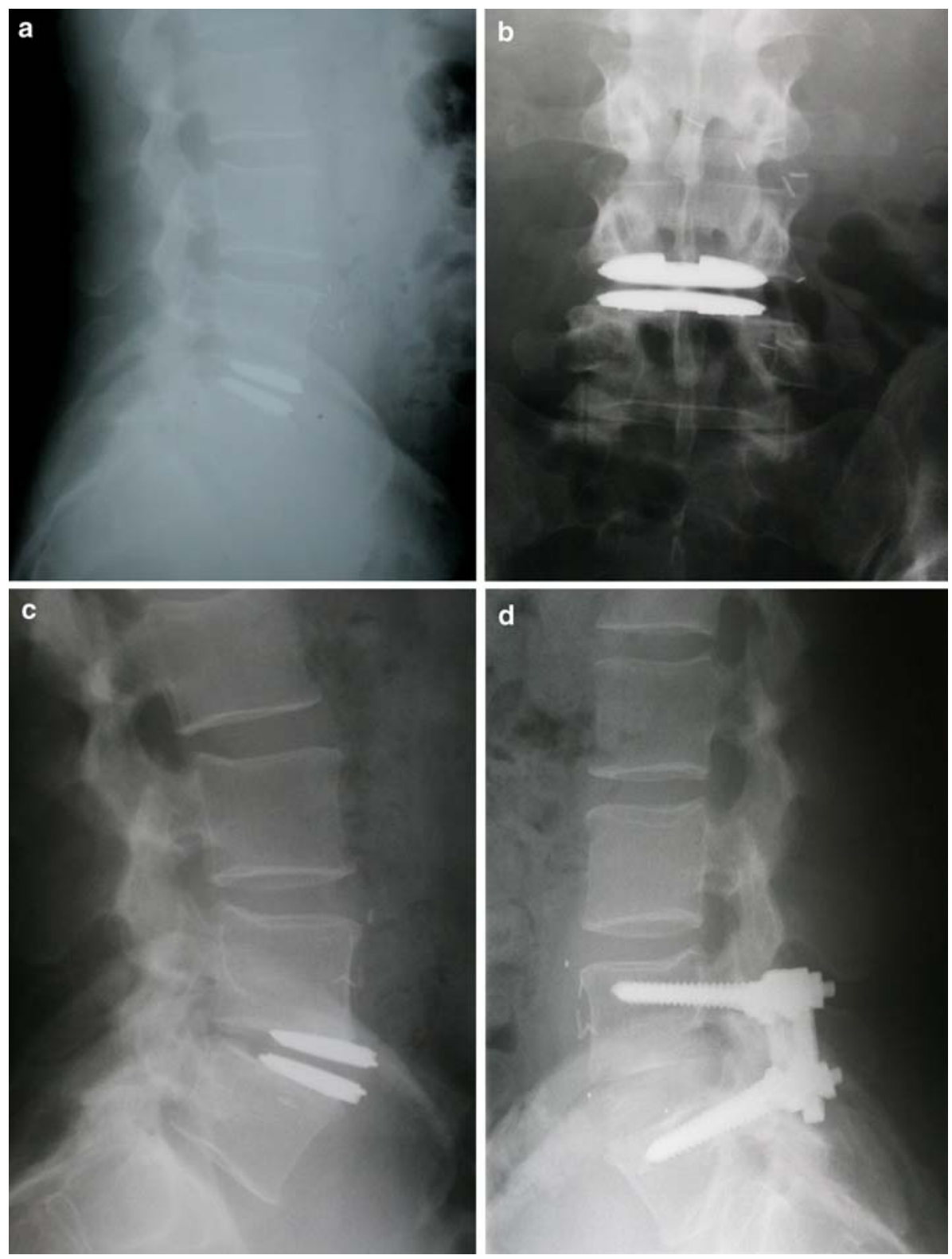

\section{Case 5}

A 44-year-old man who had long standing low back pain had a single level degenerate disc at $\mathrm{L}_{5} \mathrm{~S}_{1}$. The $\mathrm{L}_{5} \mathrm{~S}_{1}$ disc was replaced with a $29 \times 40 \times 10^{\circ} 11 \mathrm{~mm}$ AcroFlex disc. Immediately postoperatively he was well with an improvement in his symptoms. Gradual displacement of the prosthesis occurred from 3 months. The prosthesis was removed at 19 months and fusion performed using a Stabilis cage and cancellous bone graft. During surgery a tear of the left common iliac vein occurred during mobilisation this was repaired with 2.0 Prolene. Formal division of the iliac vein and artery was not necessary as the disc was removed from $\mathrm{L}_{5} \mathrm{~S}_{1}$. Preoperatively the ODI was $48 \%$ after fusion it had fallen to $10 \%$.

\section{Discussion}

The five patients reported here gained early symptomatic relief from low back and leg pain with an artificial inter-vertebral disc. However, in each case, revision following surgery for benign disc degeneration was associated with a major vascular injury. Anterior displacement of the $\mathrm{L}_{45}$ disc caused pressure on the left common iliac vein and artery with stretching of the vessels and erosion into the vessel wall in three cases. It 
was impossible to mobilise the stretched vessels from the firmly adherent surrounding dense scar tissue without vascular injury. In one case the left common iliac vein was thrombosed while in the others repairs were necessary after almost impossible mobilisation of the vessels to expose the prosthesis. Damage to the left common iliac vein and artery in one case led to both venous and arterial thrombosis. It is likely that this complication will lead to longstanding symptoms of venous insufficiency.

The only safe solution for adequate exposure of an anteriorly displaced visco-elastic artificial $\mathrm{L}_{45}$ disc is the formal division and subsequent re-anastomosis of the left common iliac vein and if necessary the left iliac artery; this strategy was successfully employed in cases 3 and 4. An anterior transperitoneal approach is essential to allow proximal and distal vessel control before division. A retroperitoneal approach in this type of revision surgery would be extremely hazardous and potentially life threatening due to an inability to control the right common iliac vein or vena cava when there is bleeding from the left common iliac vein. The aortic bifurcation would also be difficult to control.

The Link SB Charité disc is currently the most widely implanted inter-vertebral disc. This articulating disc consists of an ultra high molecular weight polyethylene spacer between two separate cobalt-chromium-molybdenum alloy plates. Griffith et al. reported displacement or dislocation of the prostheses in 6 of 139 implants with an average follow-up of 11 months. There were six reported vein injuries although it is not clear whether these injuries were associated with displacement or revision surgery or at what level the implant was placed [6]. Cinotti et al. reported only one anterior displacement of $46 \mathrm{SB}$ Charité III prostheses and no vascular injury, with a minimum follow-up of 2 years [3]. In a series of 105 patients with 154 SB Charité III discs five vascular problems were reported (two phlebitis, two pulmonary embolism and one acute leg ischaemia) although no information on the rate of revision or anterior displacement was presented in this series [10]. Zeegers et al. reported a prospective study of 50 patients where 75 SB Charité III prosthesis were inserted. None of 38 patients with full 2 year radiographic follow-up had migration of the prosthesis. One patient lost to followup had malposition and some slip but was lost to follow-up. Of 12 patients who underwent reoperation only one aortic injury was reported during failed disc re-positioning and subsequent fusion [12]. These reports are all non-randomised series without any control and in one case retrospective. The only published randomised study of inter-vertebral disc replacement compares the Charité disc with spinal fusion. This study showed that pain and disability scores are equivalent at 24 months follow-up. There was a $4.4 \%$ incidence of venous injury in the arthroplasty group and device failure in $5.1 \%$ compared to 9.1 and $2 \%$ respectively in the spinal fusion group [2].

This first design of the AcroFlex disc contained a hexane based polyolefin rubber core vulcanised between two titanium plates. A retrospective review of six patients who had AcroFlex discs, with a mean follow-up was 3.5 years, reported a poor outcome in two patients with fracture of the rubber component and persistent symptoms in a second. There were no reported cases of disc displacement or of vascular injury during one revision although the prosthesis was at $\mathrm{L}_{5} \mathrm{~S}_{1}$ [4]. Fraser et al. reported a series of 28 patients who had the third generation AcroFlex disc implanted, 17 patients had the same disc design used in this study. Eight of 28 required revision surgery for deterioration of symptoms and failure of the prosthesis. Two of the four patients who had removal of the prosthesis suffered left iliac vein injury during surgery. Fraser et al. inserted the discs through a retroperitoneal approach and suffered one anterior displacement in this case series [5]. It should be noted that there were two pilot studies. In the first series all patients were implanted in Adelaide. Displacement occurred in series 2 at both test centres but only in Manchester was displacement severe enough to warrant removal for the reasons stated. Disc displacement due to placement of the disc too far anteriorly has been reported with the PRODISC [1]. The high incidence of disc displacement in this series (5 of 13) is thought to be associated with an anterior transperitoneal approach and more extensive removal of the annulus rather than malposition. Design is also an important factor in preventing displacement since it occurred in both centres. On revision surgery no osteointegration of these implants was apparent and displacement occurred despite re-design of the endplates, as a result of the series 1 failure, to a domed surface with 4-6 tapered fins on each surface. This redesign was also inadequate.

Van Ooij has described a series of 27 patients with anterior displacement of four Charité discs one of which led to compression of the iliac artery causing symptoms of ischaemia [11]. A systematic review of total disc replacement examined the results from 564 arthroplasties in 411 patients. The majority of the cases considered were the Charité disc and only eight cases of vascular injury were identified [8]. The authors of both papers concluded that removal of artificial discs is potentially dangerous and no reliable strategy for 
revision surgery exists for a procedure without proven long-term reliability.

The five cases described in this report highlight failure of three design criteria for a disc prosthesis: (i) the rapid fixation to bone, (ii) fail-safe design to prevent damage to surrounding structure in case of failure and (iii) medium-term osteointegration [7]. If a disc does fail and needs to be removed, surgery can be hazardous with associated vascular injury [8]. Consequently, we advise that a Vascular Surgeon should be available to try and mobilise or divide and re-anastomose the left common iliac vessels when needed for adequate exposure of a $\mathrm{L}_{45}$ disc. Furthermore, this revision disc surgery is usually difficult and should be planned to include autologous blood transfusion.

\section{References}

1. Aunoble S, Donkersloot P, Le Huec JC (2004) Dislocations with intervertebral disc prosthesis: two case reports. Eur Spine J 13:464-467

2. Blumenthal S, McAfee PC, Guyer RD, Hochschuler SH, Giesler FH, Holt RT, Garcia R, Regan JJ, Ohnmeiss DD (2005) A prospective, randomised, multicenter food and drug administration investigational device exemptions study of lumbar total disc replacement with the Charite artificial disc versus lumbar fusion. Spine 30:1565-1575
3. Cinotti G, David T, Postacchini F (1996) Results of prosthesis after a minimum follow-up period of 2 years. Spine 21:995-1000

4. Enker P, Steffee A, McMillin C, Keppler L, Biscup R, Miller S (1993) Artificial disc replacement. Preliminary report with a 3-year minimum follow-up. Spine 18:1061-1070

5. Fraser RD, Ross ER, Lowery GL, Freeman BJ, Dolan M (2004) AcroFlex design and results. Spine J 4(Suppl 6):245S$251 \mathrm{~S}$

6. Griffith SL, Shelokov AP, Büttner-Janz K, LeMaire JP, Zeegers WS (1994) A multicenter retrospective study of the clinical results of the Link SB Charite intervertebral prosthesis. The initial European experience. Spine 16:1842-1849

7. Hedman TP, Kostuik JP, Fernie GR, Hellier WG (1991) Design of an intervertebral disc prosthesis. Spine 16:S256S260

8. de Kleuver M, Oner FC, Jacobs WCH (2003) Total disc replacement for chronic low back pain: background and a systematic review of the literature. Eur Spine J 12:108-116

9. Krismer M (2002) Fusion of the lumbar spine. A consideration of the indications. J Bone Joint Surg Br 84:783-794

10. Lemaire JP, Skalli W, Lavaste F, Templier A, Mendes F, Diop A, et al (1997) Intervertebral disc prosthesis results and prospects for the year 2000. Clin Orthop 337:64-76

11. Van Ooij A, Oner FC, Verbout AJ (2003) Complications of artificial disc replacement: a report of 27 patients with the SB Charité disc. J Spinal Disord Tech 16:369-383

12. Zeegers WS, Bohnen LMLJ, Laaper M, Verhaegen MJA (1999) Artificial disc replacement with the modular type SB Charite III: 2-year results in 50 prospectively studied patients. Eur Spine J 8:210-217 